\title{
INVESTIGATION ON THE DYNAMICS OF AN ON-BOARD ROTOR-BEARING SYSTEM
}

\author{
Mzaki Dakel \\ Université de Lyon, CNRS, \\ INSA-Lyon, LaMCoS UMR5259, \\ F-69621, France \\ mzaki.dakel@insa-lyon.fr
}

\author{
Sébastien Baguet \\ Université de Lyon, CNRS, \\ INSA-Lyon, LaMCoS UMR5259, \\ F-69621, France \\ sebastien.baguet@insa-lyon.fr
}

\author{
Régis Dufour \\ Université de Lyon, CNRS, \\ INSA-Lyon, LaMCoS UMR5259, \\ F-69621, France \\ regis.dufour@insa-lyon.fr
}

\section{ABSTRACT}

In ship and aircraft turbine rotors, the rotating mass unbalance and the different movements of the rotor base are among the main causes of vibrations in bending. The goal of this paper is to investigate the dynamic behavior of an onboard rotor under rigid base excitations. The modeling takes into consideration six types of base deterministic motions (rotations and translations) when the kinetic and strain energies in addition to the virtual work of the rotating flexible rotor components are computed. The finite element method is used in the rotor modeling by employing the Timoshenko beam theory. The proposed on-board rotor model takes into account the rotary inertia, the gyroscopic inertia, the shear deformation of shaft as well as the geometric asymmetry of shaft and/or rigid disk. The Lagrange's equations are applied to establish the differential equations of the rotor in bending with respect to the rigid base which represents a noninertial reference frame. The linear equations of motion display periodic parametric coefficients due to the asymmetry of the rotor and time-varying parametric coefficients due to the base rotational motions. In the proposed applications, the rotor mounted on rigid/elastic bearings is excited by a rotating mass unbalance associated with sinusoidal vibrations of the rigid base. The dynamic behavior of the rotor is analyzed by means of orbits of the rotor as well as fast Fourier transforms (FFTs).

\section{INTRODUCTION}

The rotating machines are among the vital parts of modern industrial domains and the rotor forms their key component. Base excitations of rotating machines can be caused by a machine carried on a base affected by ground movement or onboard moving systems. Rotors mounted on transportation systems are mainly excited by the rotating mass unbalance and the various motions of their base which can increase their lateral vibration and create a dynamic instability phenomenon.

The rotors are complex systems and their analysis is also complex. Many studies concentrated on making a clear understanding of the dynamics of symmetric/asymmetric rigid/flexible rotor systems supported by linear/nonlinear elastic bearings in the case of fixed base because these systems can be introduced in the modern industry [1,2]. Berlioz et al. [3] observed theoretically and experimentally the lateral instabilities of a drill-string parametrically excited under rotating conditions. Nandi and Neogy [4] presented an efficient analysis of stability for finite element models of asymmetric rotors and investigated whether an unstable rotor could be stabilized using an isotropic viscous damper.

Samali et al. [5] used the Monte Carlo simulation to simulate the nonstationary earthquake ground motions and to determine the statistics of rotating machinery response. In [6], a seismic response of a Jeffcott rotor supported by oil film bearings to a real seismic wave was examined and its stability was investigated by calculating the loci of the disk and journal centers. Bachelet et al. [7] investigated the dynamic behavior of an asymmetric rotor excited by a base seismic translation and developed an original spectral approach in order to approximate the rotor response. Da Silva Tuckmantel et al. [8] represented the supporting structure (foundation) of a rotating system by coupled as well as uncoupled modes. They found that there were limitations in identifying the vibratory modes and their corresponding damping factors in the system response. Kang et al. [9] and Cavalca et al. [10] studied the effect of the flexible foundation on the dynamic characteristics of the rotor-bearing systems.

The number of works dealing with the investigation of dynamic behavior of a rotor during a harmonic motion of its base is quite low. Duchemin et al. [11] applied the method of 
multiple scales to a simple rotor model under a base sinusoidal rotation in order to observe its stability. They presented experimental results in order to validate the analytical study. Driot et al. [12] described the orbits of a rotor induced by a base harmonic rotational movement using numerical methods. From their work, the comparison between numerical results and experimental investigation was quite satisfactory. El-Saeidy and Sticher [13] obtained the responses of a rigid rotor-bearing system subjected to rotating mass unbalance plus base harmonic excitations along or around lateral directions using analytical solutions in the case of linear bearing and a time integration scheme in the case of bearing cubic nonlinearity. Das et al. [14] performed a numerical simulation of a flexible rotor system excited by mass unbalance and rotational periodic motion of the base in order to investigate its active vibration control. This control was successful for avoiding the lateral parametric instability due to the sinusoidal rotation of the base.

Among all the literature mentioned above, there are references studying base-excited rotor systems and whose few works deal with the harmonically excited on-board rotors [11-14]. Moreover, these references concentrate on the investigation of dynamic behavior of either simple rotors (for example, Jeffcott rotor [6] and rotors modeled using the Rayleigh-Ritz method [11,12]), rotors supported by elastic bearings with constant damping and stiffness coefficients (for example, $[13,14]$ ) or rotors excited by simple motions (rotation or translation) of the base. As a consequence, the applications proposed in these works are not suitable for realistic ones. In this paper, an improved model is presented. Namely, an asymmetric rotor is discretized using the finite element method based on the Timoshenko beam theory, mounted on hydrodynamic bearings linearized with damping and stiffness coefficients calculated using the Reynolds equation [15] and excited by base combined motions (six types of deterministic rotational and translational motions). The rotary inertia, the gyroscopic inertia, the shear deformation of shaft as well as the geometric asymmetry of shaft and/or rigid disk are taken into consideration. By computing the Rayleigh damping coefficients, the effect of rotor internal damping is included in the study. The application of the Lagrange's equations using the finite element method gives the differential equations of the rotor in bending with respect to a noninertial reference frame connected to the rotor rigid base. The linear equations of motion point out periodic parametric coefficients due to the geometric asymmetry of the rotor and time-varying parametric coefficients due to the base rotational excitations. These parametric coefficients are considered as producers of internal excitation and can create lateral dynamic instability. In the presented applications, the rotor mounted on rigid or elastic linear bearings is subjected to rotating mass unbalance plus to base sinusoidal rotation without or with base sinusoidal translation. Numerical solutions for the linear equations of vibratory motion of the on-board rotor are computed and analyzed by means of orbits of the rotor as well as fast Fourier transforms (FFTs).

\section{THEORTICAL ANALYSIS OF AN ON-BOARD ROTOR-BEARING SYSTEM}

The rotor generally consists of the disk, shaft, mass unbalance, bearing and base. This section includes formulations for the rotor components and the equations of motion.

\subsection{Preliminary Calculations}

Three principal coordinate systems shown in Fig. 1 are introduced to take into account the movement of the rotor rigid base. They are attached to the ground $R^{g}$, the rigid base $R$ and the moving rotor $R^{l}$.

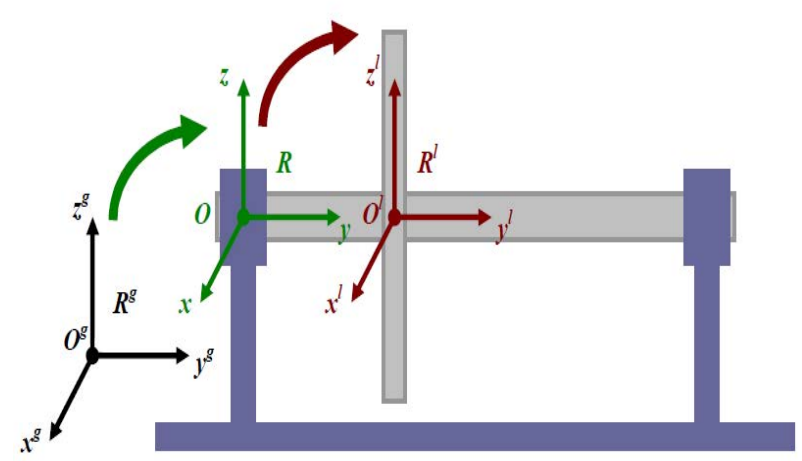

Figure 1. REFERENCE FRAMES OF THE ON-BOARD ROTOR

The rotational motions of the rotor base are described by the angular velocity vector components $\omega^{x}(t), \omega^{y}(t)$ and $\omega^{z}(t)$ of the rigid base $R$ with respect to the ground $R^{g}$ projected in the frame $R$. The translational motions of the rotor base are described by the coordinates $x_{O}(t), y_{O}(t)$ and $z_{O}(t)$ of the position vector $\mathbf{O}^{\mathrm{g}} \mathbf{O}$ projected in the frame attached to the base $R$. The Euler angles $\psi(y, t), \theta(y, t)$ and $\phi(t)$ (Fig. 2) permit describing the orientation of the rotor $R^{l}$ with respect to its base $R$. The angular velocity vector of the rotor $R^{l}$ with respect to the ground $R^{g}$ measured in the frame $R^{l}$ is given by

$$
\boldsymbol{\omega}_{R^{l}}^{R^{g}}=\boldsymbol{\omega}_{R}^{R^{g}}+\boldsymbol{\omega}_{R^{l}}^{R}=\left\langle\omega^{x^{l}}, \omega^{y^{l}}, \omega^{z^{z^{l}}}\right\rangle_{R^{l}}^{T}
$$

where $T$ is the matrix transposition symbol. The components $\left(\omega^{x^{l}}, \omega^{y^{l}}, \omega^{z^{l}}\right)$ are formulated as a function of $(\psi, \theta, \phi)$ and their time derivative as well as $\left(\omega^{x}, \omega^{y}, \omega^{z}\right)$. The rotor is supposed to rotate at a constant speed $\Omega$. So the spinning angle $\phi$ is replaced by $\Omega t$ and its derivative $\dot{\phi}$ by $\Omega(\bullet$ refers to the differentiation with respect to time $t$ ). Let us consider a generic point $C^{0}$ on the elastic line of the nondeformed shaft. Its coordinates in the frame $R$ are $(0, y, 0)$. The translational displacements $u(y, t)$ and $w(y, t)$ of the point $C^{0}$ due to bending of the flexible rotor are expressed respectively with respect to the $O x$ and $O z$ axes of the coordinate system $R$. 


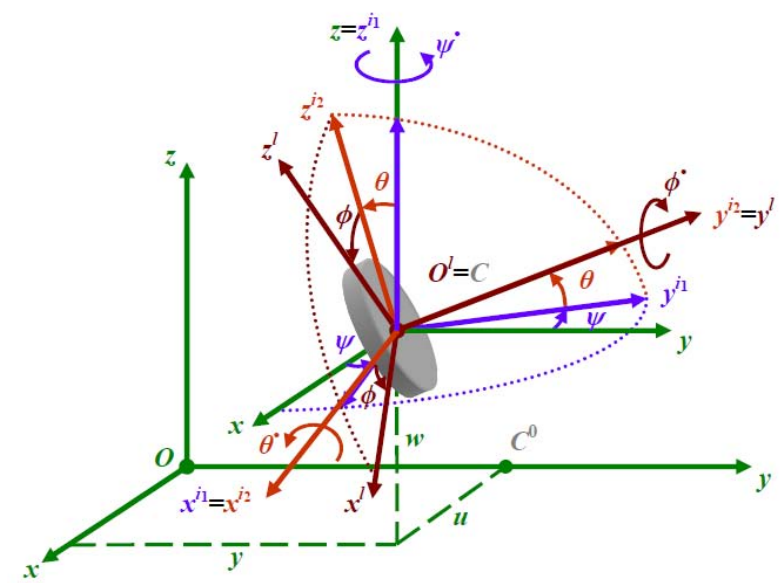

Figure 2. EULER ANGLES

\subsection{Energy and Virtual Work Calculations}

The kinetic and strain energies are measured from the ground and their terms are written with respect to the frame linked with the rotor rigid base $R$.

Disk. The disk is assumed to be rigid. Therefore only its kinetic energy $T_{d}$ is calculated as follows $[7,11]$

$$
T_{d}=\frac{m_{d}}{2}\left(\mathbf{v}_{O^{l}}^{R^{g}}\right)^{T} \mathbf{v}_{O^{\prime}}^{R^{g}}+\frac{1}{2}\left(\boldsymbol{\omega}_{R^{l}}^{R^{g}}\right)^{T} \mathbf{I}_{m_{d}} \boldsymbol{\omega}_{R^{l}}^{R^{g}}
$$

with

$$
\mathbf{I}_{m_{d}}=\operatorname{diag}\left[\begin{array}{lll}
I_{m_{d}}^{x} & I_{m_{d}}^{y} & I_{m_{d}}^{z}
\end{array}\right]=\operatorname{diag}\left[\begin{array}{lll}
I_{m_{d}}^{m o}+I_{m_{d}}^{d i} & I_{m_{d}}^{y} & I_{m_{d}}^{m o}-I_{m_{d}}^{d i}
\end{array}\right]
$$

where $m_{d}$ is the mass of the disk, $\mathbf{v}_{O^{l}}^{R^{g}}$ is the translational velocity vector of its center and $\mathbf{I}_{m_{d}}$ is its principal inertia tensor. $I_{m_{d}}^{x}, I_{m_{d}}^{y}$ and $I_{m_{d}}^{z}$ are the inertias of the disk mass about the $O x, O y$ and $O z$ axes respectively. In addition, $I_{m_{d}}^{m o}$ and $I_{m_{d}}^{d i}$ are used to explain the effects due to the mean inertia of the disk mass and those due to the inertia modeling the disk geometric asymmetry. Thus when the disk is asymmetric, $I_{m_{d}}^{x}$ as well as $I_{m_{d}}^{z}$ are different and the contribution of $I_{m_{d}}^{d i}$ in them is not nil. The translational velocity vector components $\dot{u}_{O^{l}}$, $\dot{v}_{O^{l}}$ and $\dot{w}_{O^{l}}$ of the disk center are functions of the components $\left(\omega^{x}, \omega^{y}, \omega^{z}\right)$ and the coordinates $\left(x_{O}, y_{O}, z_{O}\right)$. The final expression of the kinetic energy of the disk having a mass center positioned at the arbitrary abscissa $y_{d}$ relative to the frame $R$ is then of the following form [11]

$$
\begin{aligned}
T_{d} & =\frac{m_{d}}{2}\left(\dot{u}_{O^{\prime}}^{2}+\dot{v}_{O^{\prime}}^{2}+\dot{w}_{O^{\prime}}^{2}\right) \\
& +\frac{1}{2}\left(I_{m_{d}}^{m o}\left(\omega^{x^{l} 2}+\omega^{z^{l^{2}}}\right)+I_{m_{d}}^{y} \omega^{y^{\prime} 2}+I_{m_{d}}^{d i}\left(\omega^{x^{l} 2}-\omega^{z^{l} 2}\right)\right)
\end{aligned}
$$

Shaft. The shaft is supposed to be flexible. Therefore it is characterized by the kinetic and strain energies and modeled by beam elements. The kinetic energy of a shaft can be obtained by considering a shaft elementary volume which can be considered as a disk of thickness $d y$. Thus the kinetic energy of a shaft takes the following form $[7,11]$

$$
\begin{aligned}
& T_{s h}=\frac{\rho_{s h} S_{s h}}{2} \int_{0}^{l_{s h}}\left(\dot{u}_{O^{l}}^{2}+\dot{v}_{O^{l}}^{2}+\dot{w}_{O^{l}}^{2}\right) d y \\
& +\frac{1}{2}\left(\rho_{s h} I_{S_{s h}^{o}}^{l_{s h}} \int_{0}^{l_{s h}}\left(\omega^{x^{l} 2}+\omega^{z^{l} 2}\right) d y+2 \rho_{s h} I_{S_{s h}}^{m o} \int_{0}^{l_{s h}} \omega^{y^{\prime} 2} d y\right. \\
& \left.+\rho_{s h} I_{S_{s h}}^{d l_{s h}} \int_{0}^{l_{s h}}\left(\omega^{x^{l} 2}-\omega^{z^{l} 2}\right) d y\right)
\end{aligned}
$$

where $\rho_{s h}, S_{s h}$ and $l_{s h}$ are respectively the density, the crosssection and the length of the shaft. $I_{S_{s h}}^{m o}$ and $I_{S_{s h}}^{d i}$ are respectively the mean inertia of the cross-section and the inertia characterizing the asymmetry of the shaft. The rigid base motion relative to the ground has no influence on the strain energy of the shaft because this energy depends only on the stresses and therefore on the transverse deflection of the shaft with respect to the rotor base $R$. In addition to the bending deformation, the shear effects highlighted by Timoshenko and the geometric stiffening effects corresponding to the centrifugal stressing due to the base rotational motions are taken into account. The strain energy of a shaft is defined by

$$
\begin{aligned}
& U_{s h}=\frac{E_{s h} I_{S h} I_{S h} \int_{0}^{l_{s t}}}{2}\left(\left(\frac{\partial \psi}{\partial y}\right)^{2}+\left(\frac{\partial \theta}{\partial y}\right)^{2}\right) d y \\
& +\frac{G_{s h} k_{s h}^{m o} S_{s h}}{2} \int_{0}^{l_{b}}\left(\left(\frac{\partial u}{\partial y}+\psi\right)^{2}+\left(\frac{\partial w}{\partial y}-\theta\right)^{2}\right) d y \\
& -\frac{1}{2}\left(E_{s h} I_{S_{s h}}^{d i l} \int_{0}^{l_{l h}}\left(\left(\frac{\partial \psi}{\partial y}\right)^{2}-\left(\frac{\partial \theta}{\partial y}\right)^{2}\right) d y\right. \\
& \left.-G_{s h} k_{s h}^{d i} S_{s h} \int_{0}^{l_{t h}}\left(\left(\frac{\partial u}{\partial y}+\psi\right)^{2}-\left(\frac{\partial w}{\partial y}-\theta\right)^{2}\right) d y\right) \cos (2 \Omega t) \\
& -\left(E_{s h} I_{s i n}^{d i} \int_{0}^{l_{h}} \frac{\partial \psi}{\partial y} \frac{\partial \theta}{\partial y} d y-G_{s h} k_{s h}^{d i} S_{s h} \int_{0}^{l_{h}}\left(\frac{\partial u}{\partial y}+\psi\right)\left(\frac{\partial w}{\partial y}-\theta\right) d y\right) \sin (2 \Omega t) \\
& +\frac{\rho_{s h} S_{s h}}{4} \int_{0}^{l . t}\left(l_{s h}^{2}-y^{2}\right)\left(\left(\frac{\partial u}{\partial y}\right)^{2}+\left(\frac{\partial w}{\partial y}\right)^{2}\right) d y\left(\omega^{x^{2}}+\omega^{22}\right)
\end{aligned}
$$

where $E_{s h}$ and $G_{s h}$ are respectively the Young's modulus and the shear modulus of the shaft $\left(G_{s h}=E_{s h} /\left(2\left(1+v_{s h}\right)\right)\right.$ hence $v_{s h}$ is the Poisson's ratio). $k_{s h}^{m o}$ and $k_{s h}^{d i}$ are respectively the mean shear correction factor of the cross-section and that relative to the section asymmetry of the shaft.

Mass Unbalance. Let us consider a concentrated mass unbalance $m_{m u}$ placed at a point $P_{m u}$ on the disk $\left(y_{m u}=y_{d}\right)$ with a distance $r_{m u}$ from the shaft geometric center. Its initial angle 
with the $O z$ axis of the frame $R$ at rest is $\eta_{m u}$. The mass unbalance is characterized by the kinetic energy computed as follows [11]

$$
T_{m u}=\frac{m_{m u}}{2}\left(\mathbf{v}_{P_{m u}}^{R^{g}}\right)^{T} \mathbf{v}_{P_{m u}}^{R^{g}}
$$

The components of the mass unbalance translational velocity vector $\mathbf{v}_{P_{m u}}^{R^{g}}$ are functions of $\left(\omega^{x}, \omega^{y}, \omega^{z}\right)$ and $\left(x_{O}, y_{O}, z_{O}\right)$.

Bearing. Figure 3 shows a simple representation of a hydrodynamic bearing which is composed of a fixed journal containing a rotating shaft. The points $O$ and $O^{l}=C_{\text {sep }}$ are respectively the bearing center and the shaft geometric center. The radius, length and clearance of the bearing are respectively $r_{b e}, l_{b e}$ and $c_{b e}=r_{b e}-r_{s h}$ where $r_{s h}$ is the shaft radius. At a constant speed of rotation $\Omega$ of the rotor and for a constant static load $W_{r}$ created by the rotor weight, the shaft geometric center $C_{s e p}$ in the bearing holds a static equilibrium position defined by the displacement vector $\boldsymbol{\delta}_{b e, s e p}=\left\langle u_{b e, s e p}, w_{b e, s e p}\right\rangle_{R}{ }^{T}$ expressed in the frame $R$ or equivalently by the eccentricity $e_{b e}=\left\|\boldsymbol{\delta}_{b e, s e p}\right\|$ of the shaft center in the journal and the attitude angle $\varphi_{b e}$ between the $W_{r}$ load direction and the line of centers $\mathbf{O C}$ sep.

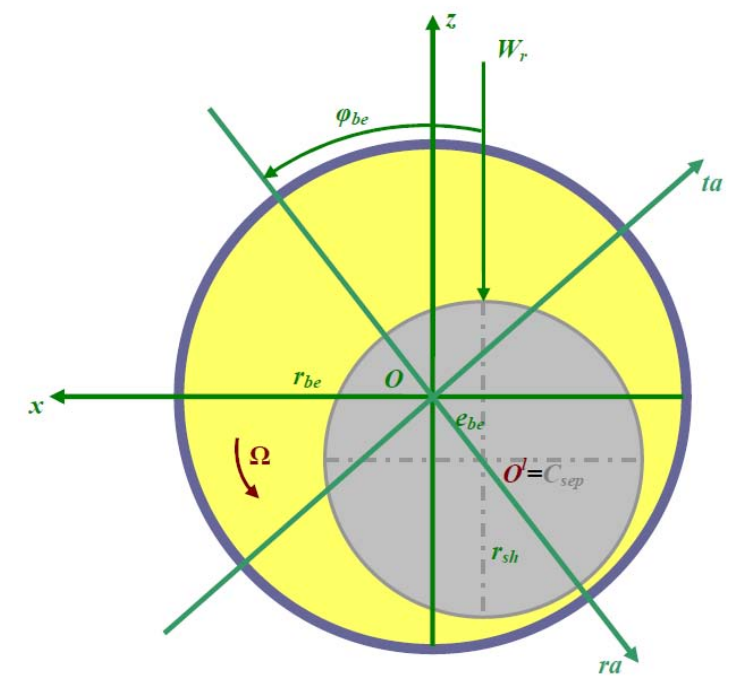

Figure 3. SCHEMATIC VIEW OF A HYDRODYNAMIC BEARING

In the present study, the short bearing theory is considered $\left(l_{b e} / d_{b e} \leq 1 / 8\right.$ where $\left.d_{b e}=2 r_{b e}\right)$ and the static solution can be reached using the following relations obtained from the Reynolds equation with the Gümbel boundary conditions [15]

$$
W_{r}=\mu r_{b e}\left(\frac{r_{b e}}{c_{b e}}\right)^{2}\left(\frac{l_{b e}}{d_{b e}}\right)^{2} l_{b e} \Omega \frac{\varepsilon_{b e}\left(16 \varepsilon_{b e}^{2}+\pi^{2}\left(1-\varepsilon_{b e}^{2}\right)\right)^{\frac{1}{2}}}{\left(1-\varepsilon_{b e}^{2}\right)^{2}}
$$

$$
\operatorname{tg}\left(\varphi_{b e}\right)=\frac{\pi}{4} \frac{\sqrt{1-\varepsilon_{b e}^{2}}}{\varepsilon_{b e}}
$$

where $\varepsilon_{b e}$ is the relative eccentricity $\left(\varepsilon_{b e}=e_{b e} / c_{b e}\right)$ and $\mu$ is the fluid film dynamic viscosity. The nonlinear Eq. (8) is solved by an iterative Newton-Raphson method and provides the eccentricity $\varepsilon_{b e}$ and then the static radial displacement $e_{b e}$ of the shaft center. The components of the vector $\boldsymbol{\delta}_{b e, s e p}$ are obtained by a classical change of basis. The hydrodynamic fluid forces $\mathbf{F}_{b e}=\left\langle F_{b e}{ }^{u}, F_{b e}{ }^{w}\right\rangle_{R}{ }^{T}$ produced by the bearings and projected in the frame $R$ can be obtained by integration of the fluid film pressure (Reynolds equation in the dynamic regime) over the bearing. In order to apply the Lagrange's equations, the virtual work $\delta W_{b e}$ of these forces has to be established

$$
\delta W_{b e}=\mathbf{F}_{b e}^{T}\left(\boldsymbol{\delta}_{b e}, \dot{\boldsymbol{\delta}}_{b e}\right) \delta \boldsymbol{\delta}_{b e}
$$

If the transverse dynamic displacements $\boldsymbol{\delta}_{b e}=\left\langle u_{b e}, w_{b e}\right\rangle_{R}^{T}$ of the shaft elastic line are supposed to be small in the vicinity of the static position $\boldsymbol{\delta}_{b e \text { sep }}$, the linear analysis of bearings can be applied by constructing a first order Taylor expansion of the fluid film forces $\mathbf{F}_{b e}\left(\boldsymbol{\delta}_{b e}, \dot{\boldsymbol{\delta}}_{b e}\right)$ in the vicinity of the static hydrodynamic forces $\mathbf{F}_{b e}\left(\boldsymbol{\delta}_{b e, s e p}, \mathbf{0}\right)$ as follows

$$
\mathbf{F}_{b e}\left(\boldsymbol{\delta}_{b e}, \dot{\boldsymbol{\delta}}_{b e}\right)=\mathbf{F}_{b e}\left(\boldsymbol{\delta}_{b e, s e p}, \mathbf{0}\right)-\mathbf{c}_{b e} \Delta \dot{\boldsymbol{\delta}}_{b e}-\mathbf{k}_{b e} \Delta \boldsymbol{\delta}_{b e}
$$

with

$$
\begin{gathered}
\mathbf{c}_{b e}=\left[\begin{array}{ll}
c_{b e}^{x x} & c_{b e}^{x z} \\
c_{b e}^{z x} & c_{b e}^{z z}
\end{array}\right] ; \quad \mathbf{k}_{b e}=\left[\begin{array}{ll}
k_{b e}^{x x} & k_{b e}^{x z} \\
k_{b e}^{z x} & k_{b e}^{z z}
\end{array}\right] \\
\Delta \boldsymbol{\delta}_{b e}=\boldsymbol{\delta}_{b e}-\boldsymbol{\delta}_{b e, s e p} \quad ; \quad \Delta \dot{\boldsymbol{\delta}}_{b e}=\dot{\boldsymbol{\delta}}_{b e}
\end{gathered}
$$

where $\mathbf{c}_{b e}$ and $\mathbf{k}_{b e}$ are the damping and stiffness matrices of the linearized hydrodynamic bearing (Fig. 4) whose analytical expressions can be found in [15].

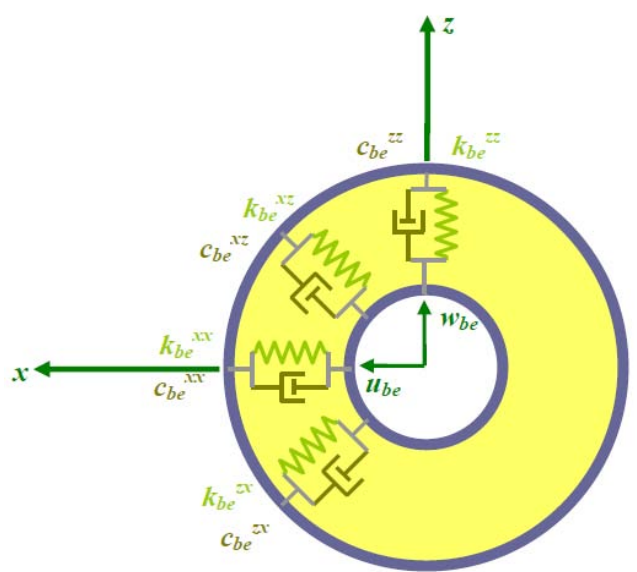

Figure 4. BEARING DAMPING AND STIFFNESS COEFFICIENTS 


\subsection{Equations of Motion}

The finite element method is chosen for discretizing the rotor and describing its flexural motion as a function of the nodal displacement vector defined by $\boldsymbol{\delta}^{n}=\left\langle u^{n}, w^{n}, \theta^{n}, \psi^{n}\right\rangle_{R}{ }^{T}$ because the rotor has two lateral translations and two rotations at each node. The finite element used for the shaft modeling has two nodes and the shape functions are based on the Timoshenko beam theory.

The linear equations of motion of the finite element rotor model are obtained after applying the Lagrange's equations to the energies for the disk, the shaft finite elements and the mass unbalance as well as to the virtual work of the hydrodynamic bearings and assembling appropriately the produced matrices and vectors. They are written with respect to the noninertial reference frame connected to the rotor rigid base $R$ as follows

$$
\begin{aligned}
\mathbf{M}_{r}(t) \ddot{\boldsymbol{\delta}}_{r}+\mathbf{C}_{r}(t) \dot{\boldsymbol{\delta}}_{r}+\mathbf{K}_{r}(t) \boldsymbol{\delta}_{r}= & \mathbf{F}_{r}(t) \\
& +\mathbf{F}_{b e}\left(\boldsymbol{\delta}_{r, s e p}, \mathbf{0}\right)+\mathbf{K}_{b e} \boldsymbol{\delta}_{r, s e p}
\end{aligned}
$$

with $\mathbf{M}_{r}(t), \mathbf{C}_{r}(t)$ and $\mathbf{K}_{r}(t)$ the mass, damping and stiffness matrices with time-varying parameters due to the asymmetry of the rotating rotor and to its moving base. $\ddot{\boldsymbol{\delta}}_{r}, \dot{\boldsymbol{\delta}}_{r}$ and $\boldsymbol{\delta}_{r}$ are respectively the acceleration, velocity and displacement vectors of the on-board rotor-bearin system of dimension $4\left(n_{e s h}+1\right) \times 1$ hence $n_{e s h}$ is the number of shaft finite elements. $\mathbf{F}_{r}(t)$ is the external force vector. In addition, the static hydrodynamic force vector $\mathbf{F}_{b e}\left(\boldsymbol{\delta}_{r, \text { sep }}, \mathbf{0}\right)$ of the bearings is opposite and equal to the rotor weight vector $\mathbf{F}_{d, s h}^{W_{r}}$. Lastly, $\boldsymbol{\delta}_{r, s e p}$ is the static solution vector of the rotor due to the hydrodynamic bearings.

The matrices of Eq. (14) are expressed in what follows

$$
\begin{aligned}
\mathbf{M}_{r}(t) & =\mathbf{M}_{d, s h}+\mathbf{M}_{d, s h}^{c_{2}} \cos (2 \Omega t)+\mathbf{M}_{d, s h}^{s_{2}} \sin (2 \Omega t) \\
\mathbf{C}_{r}(t) & =\mathbf{C}_{b e}+\mathbf{C}_{d, s h}^{i d}+\mathbf{C}_{d, s h}^{g} \Omega+\mathbf{C}_{d, s h}^{g, c_{2}} \Omega \cos (2 \Omega t) \\
& +\mathbf{C}_{d, s h}^{g, s_{2}} \Omega \sin (2 \Omega t)+\mathbf{C}_{d, s h, b}^{r e, \omega^{y}} \omega^{y} \\
\mathbf{K}_{r}(t)= & \mathbf{K}_{b e}+\mathbf{K}_{s h}^{e}+\mathbf{K}_{s h}^{e, c_{2}} \cos (2 \Omega t)+\mathbf{K}_{s h}^{e, s_{2}} \sin (2 \Omega t) \\
& +\mathbf{K}_{d, s h, b}^{r e, \dot{\omega}^{y}} \dot{\omega}^{y}+\mathbf{K}_{d, s h, b}^{r e, \Omega \omega^{y}} \Omega \omega^{y}+\left(\mathbf{K}_{d, s h, b}^{r e, \omega^{x 2}}+\mathbf{K}_{d, s h, b}^{g s e, \omega^{r 2}}\right) \omega^{x 2} \\
& +\mathbf{K}_{d, s h, b}^{r e, \omega^{y 2}} \omega^{y 2}+\left(\mathbf{K}_{d, s h, b}^{r e, \omega^{22}}+\mathbf{K}_{d, s h, b}^{g s e, \omega^{22}}\right) \omega^{z 2}+\mathbf{K}_{d, s h, b}^{r e, \omega^{x} \omega^{2}} \omega^{x} \omega^{z} \\
& +\left(\mathbf{K}_{d, s h, b}^{r e, \dot{\omega}^{y}, c_{2}} \dot{\omega}^{y}+\mathbf{K}_{d, s h, b}^{r e, \Omega \omega^{y}, c_{2}} \Omega \omega^{y}+\mathbf{K}_{d, s h, b}^{r e, \omega^{x 2}, c_{2}} \omega^{x 2}\right. \\
& \left.+\mathbf{K}_{d, s h, b}^{r e, \omega^{y 2}, c_{2}} \omega^{y 2}+\mathbf{K}_{d, s h, b}^{r e, \omega^{22}, c_{2}} \omega^{z 2}+\mathbf{K}_{d, s h, b}^{r e, \omega^{x} \omega^{2}, c_{2}} \omega^{x} \omega^{z}\right) \cos (2 \Omega t) \\
& +\left(\mathbf{K}_{d, s h, b}^{r e, \omega^{y}, s_{2}} \dot{\omega}^{y}+\mathbf{K}_{d, s h, b}^{r e, \Omega \omega^{y}, s_{2}} \Omega \omega^{y}+\mathbf{K}_{d, s h, b}^{r e, \omega^{x 2}, s_{2}} \omega^{x 2}\right. \\
& \left.+\mathbf{K}_{d, s h, b}^{r e, \omega^{y, 2}, s_{2}} \omega^{y 2}+\mathbf{K}_{d, s h, b}^{r e, \omega^{x} \omega^{z}, s_{2}} \omega^{x} \omega^{z}\right) \sin (2 \Omega t)
\end{aligned}
$$

The subscripts " $d$ ", "sh", "be" and " $b$ " refer to the disk, shaft, bearing as well as base respectively and express the contribution to the phenomenon represented by the corresponding matrix. The superscripts " $c_{2}$ " and " $s_{2}$ " denote the geometric asymmetry of the rotor expressed in terms of the time-varying trigonometric functions $\cos (2 \Omega t)$ and $\sin (2 \Omega t)$. The superscript " $i d$ " stands for the rotor internal damping introduced by estimating the Rayleigh damping coefficients, " $g$ " for the rotor gyroscopic effect, " $e$ " for the shaft elasticity corresponding to the bending and shear deformations, " $r e$ " for the rotational effects due to the base rotations (these effects come from the kinetic energies of the disk and the shaft) and "gse" for the geometric stiffening effects corresponding to the centrifugal stress due to the base rotations.

The vector $\mathbf{F}_{r}(t)$ is defined as follows

$$
\begin{aligned}
\mathbf{F}_{r}(t) & =\mathbf{F}_{d, s h}^{W_{r}}+\mathbf{F}_{m u}(t)+\mathbf{F}_{m u, b}(t)+\mathbf{F}_{d, s h, b}(t)+\mathbf{F}_{d, s h, b}^{c_{2}}(t) \cos (2 \Omega t) \\
& +\mathbf{F}_{d, s h, b}^{s_{2}}(t) \sin (2 \Omega t) \\
& =-\mathbf{V}_{d, s h}^{W_{r}} W_{r}+\mathbf{V}_{m u}^{c_{1}} \Omega^{2} \cos (\Omega t)+\mathbf{V}_{m u}^{s_{1}} \Omega^{2} \sin (\Omega t) \\
& +\left(\mathbf{V}_{m u, b}^{\dot{\omega}^{y}, c_{1}} \dot{\omega}^{y}+\mathbf{V}_{m u, b}^{\Omega \omega^{y}, c_{1}} \Omega \omega^{y}+\mathbf{V}_{m u, b}^{\omega^{x^{2}}, c_{1}} \omega^{x 2}+\mathbf{V}_{m u, b}^{\omega^{y 2}, c_{1}} \omega^{y 2}\right. \\
& \left.+\mathbf{V}_{m u, b}^{\omega^{22}, c_{1}} \omega^{z 2}+\mathbf{V}_{m u, b}^{\omega^{x} \omega^{z}, c_{1}} \omega^{x} \omega^{z}\right) \cos (\Omega t) \\
& +\left(\mathbf{V}_{m u, b}^{\dot{\omega}^{y}, s_{1}} \dot{\omega}^{y}+\mathbf{V}_{m u, b}^{\Omega \omega^{y}, s_{1}} \Omega \omega^{y}+\mathbf{V}_{m u, b}^{\omega^{x 2}, s_{1}} \omega^{x 2}\right. \\
& \left.+\mathbf{V}_{m u, b}^{\omega^{y 2}, s_{1}} \omega^{y 2}+\mathbf{V}_{m u, b}^{\omega^{2}, s_{1}} \omega^{z 2}+\mathbf{V}_{m u, b}^{\omega^{x} \omega^{z}, s_{1}} \omega^{x} \omega^{z}\right) \sin (\Omega t) \\
& -\mathbf{V}_{d, s h, b}^{u}\left(\ddot{x}_{O}+2 \dot{z}_{O} \omega^{y}-2 \dot{y}_{O} \omega^{z}+z_{O}\left(\dot{\omega}^{y}+\omega^{x} \omega^{z}\right)\right. \\
& \left.-y_{O}\left(\dot{\omega}^{z}-\omega^{x} \omega^{y}\right)-x_{O}\left(\omega^{y 2}+\omega^{z 2}\right)\right) \\
& -\mathbf{V}_{d, s h, b}^{w}\left(\ddot{z}_{O}+2 \dot{y}_{O} \omega^{x}-2 \dot{x}_{O} \omega^{y}+y_{O}\left(\dot{\omega}^{x}+\omega^{y} \omega^{z}\right)\right. \\
& \left.-x_{O}\left(\dot{\omega}^{y}-\omega^{x} \omega^{z}\right)-z_{O}\left(\omega^{x 2}+\omega^{y 2}\right)\right) \\
& -\mathbf{V}_{d, s h, b}^{y w}\left(\dot{\omega}^{x}+\omega^{y} \omega^{z}\right)+\mathbf{V}_{d, s h, b}^{y u}\left(\dot{\omega}^{z}-\omega^{x} \omega^{y}\right) \\
& -\mathbf{V}_{d, s h, b}^{\theta}\left(\dot{\omega}^{x}+\omega^{y} \omega^{z}\right)-\mathbf{V}_{d, s h, b}^{\psi}\left(\dot{\omega}^{z}-\omega^{x} \omega^{y}\right) \\
& -\mathbf{V}_{d, s h, b}^{y \psi}\left(\Omega \omega^{x}+\omega^{x} \omega^{y}\right)+\mathbf{V}_{d, s h, b}^{y \theta}\left(\Omega \omega^{z}+\omega^{y} \omega^{z}\right) \\
& -\mathbf{V}_{d, s h, b}^{\theta, c_{2}}\left(\dot{\omega}^{x}-2 \Omega \omega^{z}-\omega^{y} \omega^{z}\right) \cos (2 \Omega t) \\
& +\mathbf{V}_{d, s h, b}^{\psi, c_{2}}\left(\dot{\omega}^{z}+2 \Omega \omega^{x}+\omega^{x} \omega^{y}\right) \cos (2 \Omega t) \\
& +\mathbf{V}_{d, s h, b}^{\psi, s_{2}}\left(\dot{\omega}^{x}-2 \Omega \omega^{z}-\omega^{y} \omega^{z}\right) \sin (2 \Omega t) \\
& +\mathbf{V}_{d, s h, b}^{\theta, s_{2}}\left(\dot{\omega}^{z}+2 \Omega \omega^{x}+\omega^{x} \omega^{y}\right) \sin (2 \Omega t) \\
&
\end{aligned}
$$

where $\mathbf{V}_{m u}\left(\mathbf{F}_{m u}(t)\right), \mathbf{V}_{d, s h, b}\left(\mathbf{F}_{d, s h, b}(t)\right)$ and $\mathbf{V}_{m u, b}\left(\mathbf{F}_{m u, b}(t)\right)$ are load vectors (force vectors) associated respectively with the mass unbalance, the inertia force due to base motions and that due to coupling between both phenomena. The superscripts " $c_{1}$ " and " $s_{1}$ " signify the components of the mass unbalance force expressed in terms of the time-varying trigonometric functions $\cos (\Omega t)$ and $\sin (\Omega t)$. The superscripts $u, w, \psi$ and $\theta$ denote the direction of the action force components associated with the rotor base motions.

The transient dynamic motion of the rotor is then obtained by solving Eq. (14) by means of the implicit Newmark timestep integration algorithm based on the average acceleration. The static equilibrium position $\boldsymbol{\delta}_{r, \text { sep }}$ is used to initialize the transient dynamic problem. The final integration time is chosen 
such that the transient effects have disappeared and the steadystate regime has been reached.

\section{NUMERICAL SIMULATIONS AND DISCUSSION}

The symmetric rotor-rigid/elastic linearized bearing system presented in Fig. 5 is subjected to rotating mass unbalance as well as to base harmonic rotation without/with base harmonic translation: the rotation around the $O x$ axis is given by $\omega^{x}=\omega^{x, a} \cos \left(\Omega^{x} t\right)$ in $\mathrm{rad} / \mathrm{s}$, while the translation along the $O z$ axis is expressed as $z_{O}=Z_{O} \cos \left(\Omega^{z} t\right)$ in $\mathrm{m}$.

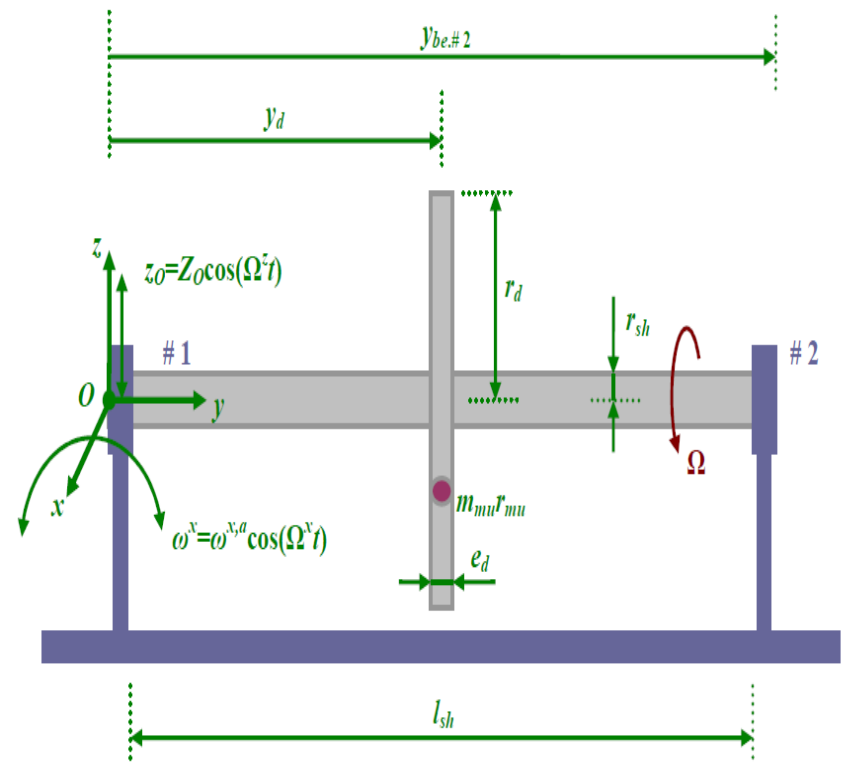

Figure 5. ON-BOARD ROTOR-BEARING SYSTEM CONFIGURATION

The physical properties as well as the geometry of the rotor and the bearings are listed in Table 1 . The shaft is discretized into eight identical 2-node Timoshenko beam finite elements. The disk is located at node 5 and the bearings \# 1 and \# 2 are respectively placed at nodes 1 and 9 . The rotor runs at a speed of rotation $\Omega=1200 \mathrm{rpm}$ ( $20 \mathrm{~Hz}=$ mass unbalance frequency).

The static equilibrium position of the shaft geometric center in the fluid film bearings is given by $\boldsymbol{\delta}_{\text {be,sep }}=<-5.71 \times 10^{-5},-1.76 \times 10^{-4}>_{R}{ }^{T} \mathrm{~m}$. The bearing damping and stiffness matrices are expressed in what follows

$$
\begin{aligned}
& \mathbf{c}_{b e}=\left[\begin{array}{ll}
3.50 \times 10^{3} & 1.08 \times 10^{4} \\
1.08 \times 10^{4} & 7.57 \times 10^{4}
\end{array}\right] \mathrm{N} / \mathrm{m} / \mathrm{s} \\
& \mathbf{k}_{b e}=\left[\begin{array}{ll}
1.30 \times 10^{6} & 1.32 \times 10^{6} \\
6.30 \times 10^{6} & 1.94 \times 10^{7}
\end{array}\right] \mathrm{N} / \mathrm{m}
\end{aligned}
$$

The rotor system is supported either by rigid bearings or hydrodynamic ones leading in the case of fixed base either to symmetric damping and stiffness matrices with isotropic diagonal and cross-coupling terms or to nonsymmetric matrices respectively.

Table 1. ROTOR AND BEARING DATA

\begin{tabular}{cc}
\hline \hline Disk material density & $7800 \mathrm{~kg} / \mathrm{m}^{3}$ \\
Disk radius & $0.15 \mathrm{~m}$ \\
Disk thickness & $0.03 \mathrm{~m}$ \\
Disk location & $0.2 \mathrm{~m}$ \\
Shaft material density & $7800 \mathrm{~kg} / \mathrm{m}^{3}$ \\
Young's modulus of the shaft & $2 \times 10^{11} \mathrm{~N} / \mathrm{m}^{2}$ \\
Poisson's ratio of the shaft & 0.3 \\
Shaft radius & $0.04 \mathrm{~m}$ \\
Shaft length & $0.4 \mathrm{~m}$ \\
Mass unbalance & $1500 \mathrm{~g} \mathrm{~mm}, 0^{\circ}$ \\
\hline Radius of the bearings & $0.04 \mathrm{~m}$ \\
Length of the bearings & $0.01 \mathrm{~m}$ \\
Locations of the bearings & $0 \mathrm{~m}, 0.4 \mathrm{~m}$ \\
Radial clearance of the bearings & $2 \times 10^{-4} \mathrm{~m}$ \\
Oil film dynamic viscosity & $288 \times 10^{-4} \mathrm{~Pa} \mathrm{~s}$ \\
\hline \hline
\end{tabular}

Figure 6 shows the classical disk orbit due to the mass unbalance for rigid bearings and a fixed base. Since the matrices of the rotor-bearing system are symmetric and skewsymmetric with isotropic diagonal and cross-coupling components, the dynamic behavior is symmetric and the orbit is circular. Its center coincides with the point $O$ (bearing center). FFT of the orbit shows that the rotor displacement has an amplitude almost of order $1 \times 10^{-7} \mathrm{~m}$ and the same mass unbalance frequency $(20 \mathrm{~Hz})$.
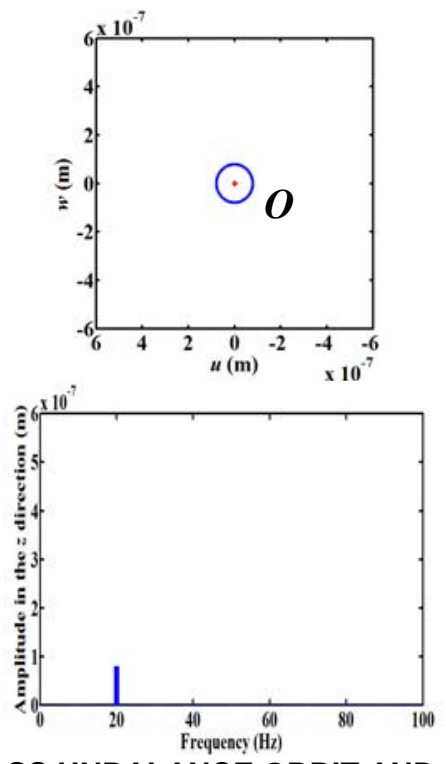

Figure 6. MASS UNBALANCE ORBIT AND ITS z-FFT AT THE DISK FOR RIGID BEARINGS AND FIXED BASE 
Figure 7 gives the disk orbit and $z$-FFT of the rotor vibrations due to the mass unbalance and the base rotation around the $O x$ axis. This rotation generates vectors corresponding to force components acting respectively in the $O x$ direction (due to the Coriolis acceleration, i.e., the term $-\mathbf{V}_{d, s h, b}^{y \psi} \Omega \omega^{x}$ in Eq. (18)) and in the $O z$ direction (due to the tangential acceleration, i.e., the the terms $-\mathbf{V}_{d, s h, b}^{y w} \dot{\omega}^{x}$ and $-\mathbf{V}_{d, s h, b}^{\theta} \dot{\omega}^{x}$ in Eq. (18)). The two transverse displacements associated with these force components are different and this breaks the asymmetry of the rotor behavior. For the selected speed of rotation of the rotor, the $z$-amplitude is very higher than the $x$-amplitude which remains mostly the same. FFT exhibits two frequency components due to the mass unbalance excitation $(20 \mathrm{~Hz})$ and to the base harmonic motion $(80 \mathrm{~Hz})$.
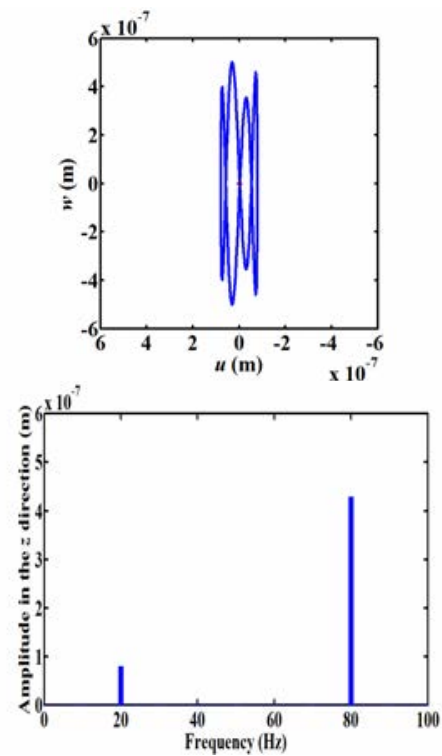

\section{Figure 7. DISK ORBIT AND ITS z-FFT FOR RIGID BEARINGS AND BASE HARMONIC ROTATION: $\omega^{x, a}=5 \times 10^{-2} \mathrm{rad} / \mathrm{s}$ AND $\Omega^{x}=80 \mathrm{~Hz}$}

Figure 8 presents the disk orbit due to the mass unbalance effect in the presence of hydrodynamic bearings. The nonsymmetric damping and stiffness coefficients make the bearings anisotropic and the orbit elliptical with diagonal axes defining the phase between the mass unbalance excitation and the rotor response. The orbit center coincides with the point $C_{\text {sep }}$ (static position of the shaft center in the bearings). It is noted that the orbit is large compared to that corresponding to rigid bearings because of the combination between the bending modes of the rotor and the rigid body modes relative to the rotor motion in the hydrodynamic bearings. $z$-FFT indicates that the disk vibration has an amplitude of about $7 \times 10^{-6} \mathrm{~m}$ and the same mass unbalance frequency $(20 \mathrm{~Hz})$.
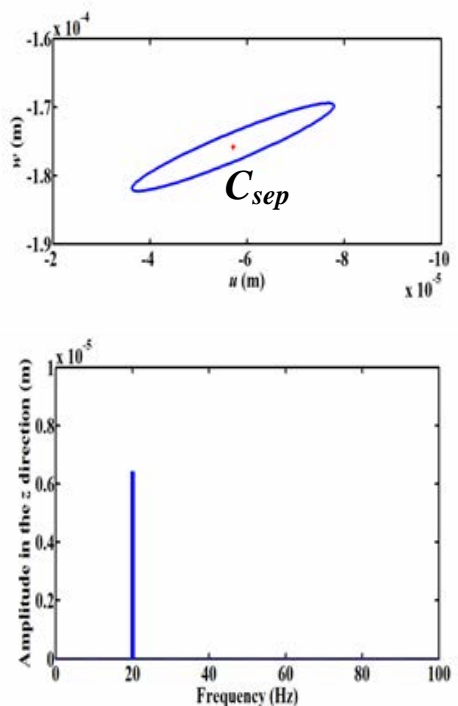

\section{Figure 8. MASS UNBALANCE ORBIT AND ITS $z$-FFT AT} THE DISK FOR FLEXIBLE BEARINGS AND FIXED BASE

Figure 9 displays the disk orbits for hydrodynamic bearings during base rotational motions. The orbit characteristics (shape and magnitude) change with amplitude and frequency of the base harmonic excitation. It should be noted that for very small amplitudes and different frequencies, the orbit shapes become more complicated with respect to those obtained when the base is fixed (see Fig. 9(a)). On the other hand, the base motion amplitudes change the $x$ and $z$ orbit magnitudes. $z$-FFTs exhibit two frequency components due to the mass unbalance excitation $(20 \mathrm{~Hz})$ and to the base harmonic motions $(50 \mathrm{~Hz}$ or $80 \mathrm{~Hz})$. They also show that the displacement amplitude due to the mass unbalance excitation $(20 \mathrm{~Hz})$ does not roughly change for base motions of frequency $50 \mathrm{~Hz}$ and $80 \mathrm{~Hz}$, i.e., the influence of the base rotations on the mass unbalance excitation (see Eq. (18)) can be neglected at the lower amplitudes of the base excitation.

Figures 10 and 11 display the orbits of the disk and their $z$-FFTs in the presence of mass unbalance forces and of a combination of base rotational and translational motions. In Fig. 10, the base rotation is kept constant $\left(\omega^{x, a}=5 \times 10^{-2} \mathrm{rad} / \mathrm{s}\right.$ and $\Omega^{x}=50 \mathrm{~Hz}$ ) while the base translation has two amplitudes and three frequencies. In Fig. 11, the base translation is kept constant $\left(Z_{O}=5 \times 10^{-5}\right.$ and $\left.\Omega^{z}=50 \mathrm{~Hz}\right)$ while the base rotation has two amplitudes and three frequencies. Increasing the amplitudes and frequencies makes the orbits larger and more complicated especially when compared with the mass unbalance orbit for a fixed base; see Fig. 8. The assumption to have linear behavior of the bearing is questionable. FFTs in Figs. 10 and 11 exhibit three frequency components due to the mass unbalance excitation $(20 \mathrm{~Hz})$, to the base harmonic rotation $(50 \mathrm{~Hz}$ in Fig. 10 and $80 \mathrm{~Hz}$ in Fig. 11) as well as to the base harmonic translation $(80 \mathrm{~Hz}$ in Fig. 10 and $50 \mathrm{~Hz}$ in Fig. 11). 


\section{CONCLUSIONS}

A finite element model is presented to analyze the dynamic behavior of a symmetric on-board rotor-rigid/hydrodynamic bearing system whose base is subjected to sinusoidal rotations without/with sinusoidal translations.

The rotational effects and the geometric stiffening effects relative to the centrifugal stressing due to the base rotations are taken into account.

The base rotations create time-varying parametric coefficients which can lead to lateral dynamic instability.

In the case of a rotor mounted on rigid bearings and running at the lower speeds of rotation, the base rotation around a transverse axis creates an orbit having its greatest magnitude in the perpendicular transverse direction. Unlike the previous case, the base rotation effects concern the two transverse directions when the rotor is supported by hydrodynamic bearings.
It is noted that the shape and the magnitude of the orbits can be significantly affected by the base motion frequency and amplitude respectively.

In the case of considerable rotation amplitude compared to the speed of rotation of the rotor, it is shown that the mass unbalance forces can depend on the base rotation around a transverse axis.

The frequency components due to the mass unbalance excitation and to the base harmonic motions appear in FFTs of the rotor flexural vibrations.

In the case of large orbits, the assumption of a linearized hydrodynamic bearing model is questionable. A nonlinear model is to be considered and the hydrodynamic bearings have to be treated as external nonlinear forces acting on the shaft within the bearings. (a) $\omega^{x, a}=10^{-2} \mathrm{rad} / \mathrm{s}$
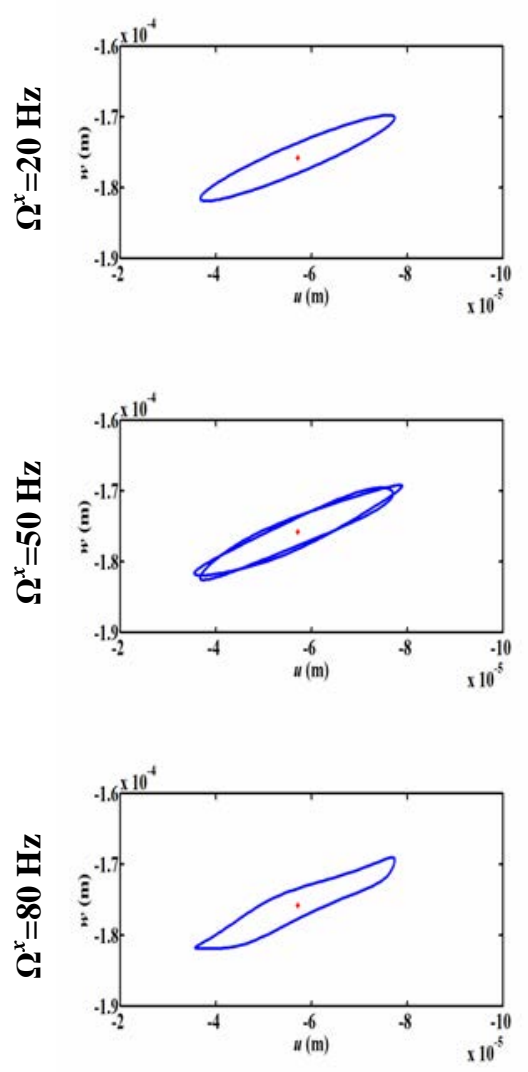
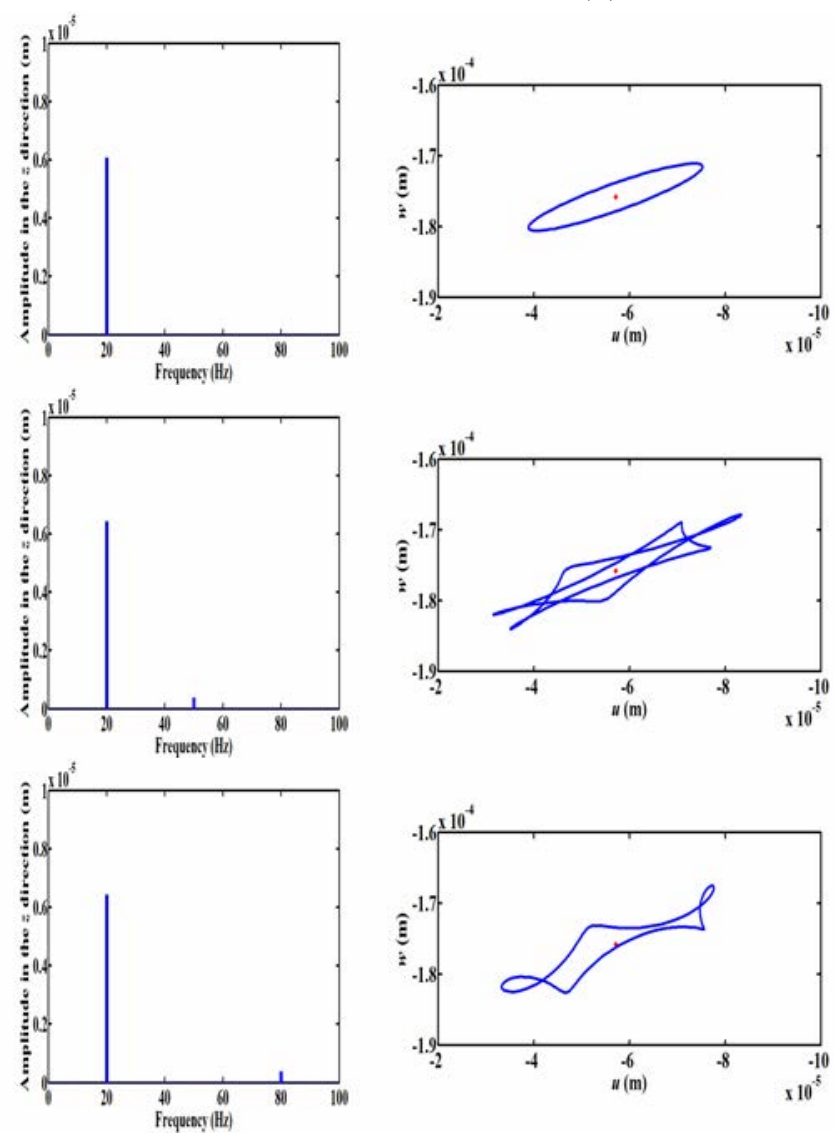

(b) $\omega^{x, a}=5 \times 10^{-2} \mathrm{rad} / \mathrm{s}$
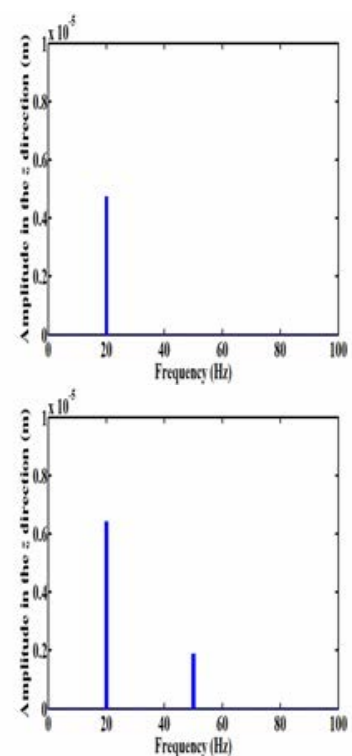

Figure 9. DISK ORBITS AND THEIR $z$-FFTS FOR FLEXIBLE BEARINGS AND TWO AMPLITUDES OF BASE HARMONIC ROTATIONS: $\omega^{x, a}=$ (a) $10^{-2} \mathrm{rad} / \mathrm{s}$, (b) $5 \times 10^{-2} \mathrm{rad} / \mathrm{s},\left(\Omega^{x}=20 \mathrm{~Hz}, 50 \mathrm{~Hz}\right.$ AND $\left.80 \mathrm{~Hz}\right)$ 
(a) $Z_{O}=10^{-5} \mathrm{~m}$
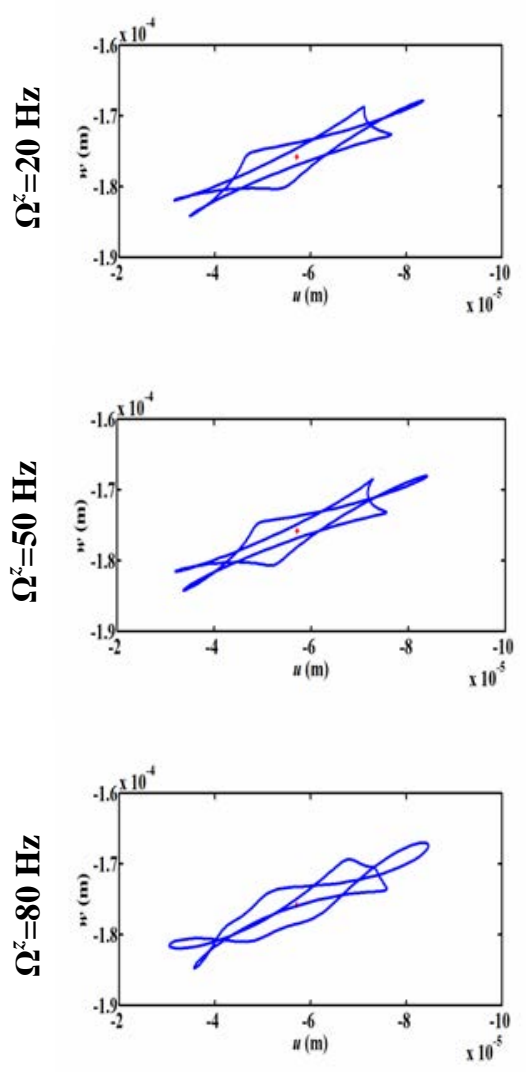
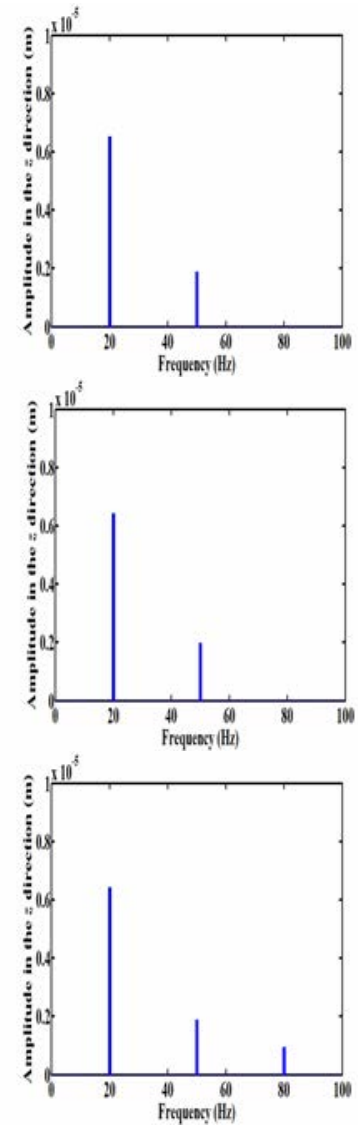

(b) $Z_{O}=5 \times 10^{-5} \mathrm{~m}$
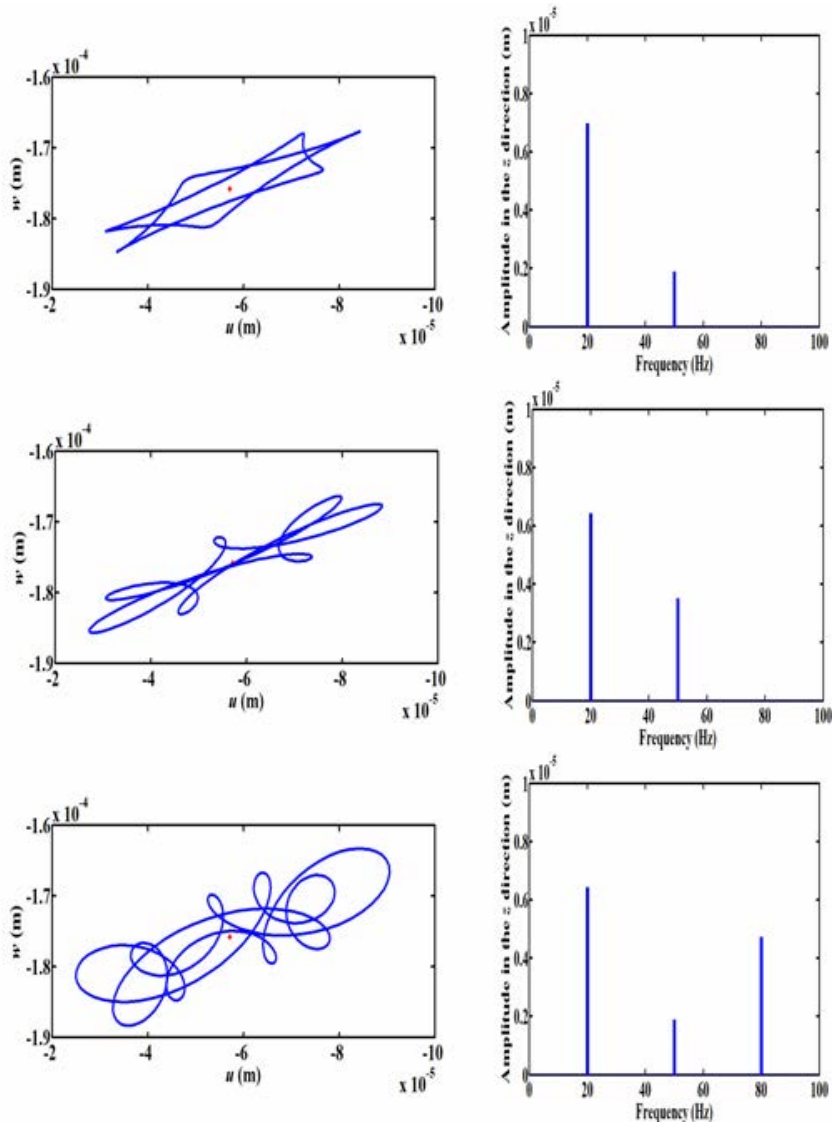

Figure 10. ORBITS AND THEIR z-FFTs AT THE DISK FOR FLEXIBLE BEARINGS AND BASE SINUSOIDAL ROTATION $\left(\omega^{x, a}=5 \times 10^{-2} \mathrm{rad} / \mathrm{s}\right.$ AND $\left.\Omega^{x}=50 \mathrm{~Hz}\right)$, COMBINED WITH TWO AMPLITUDES OF BASE SINUSOIDAL TRANSLATIONS: $Z_{O}=\left(\right.$ a) $10^{-5} \mathrm{~m},\left(\right.$ b) $5 \times 10^{-5} \mathrm{~m},\left(\Omega^{z}=20 \mathrm{~Hz}, 50 \mathrm{~Hz}\right.$ AND $\left.80 \mathrm{~Hz}\right)$

(a) $\omega^{x, a}=10^{-2} \mathrm{rad} / \mathrm{s}$
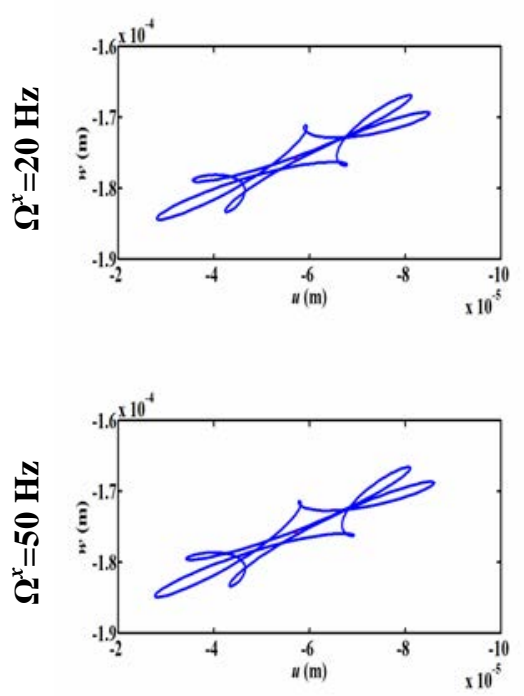

(b) $\omega^{x, a}=5 \times 10^{-2} \mathrm{rad} / \mathrm{s}$
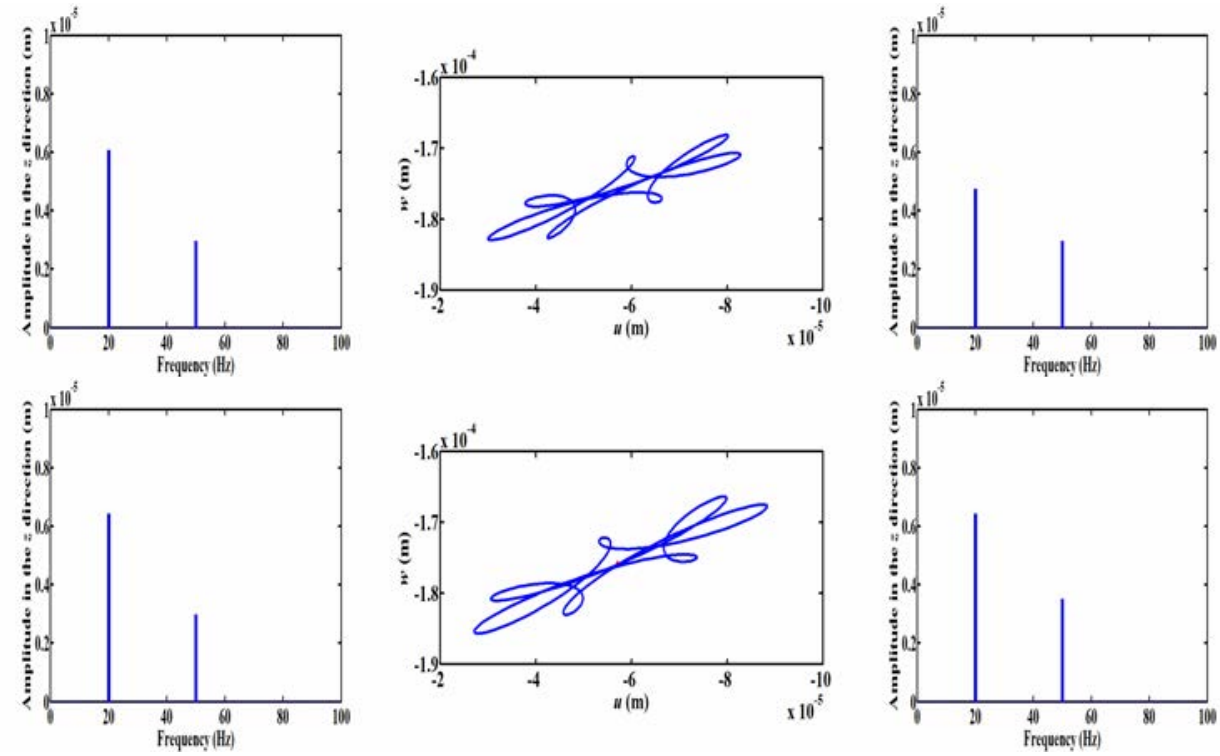

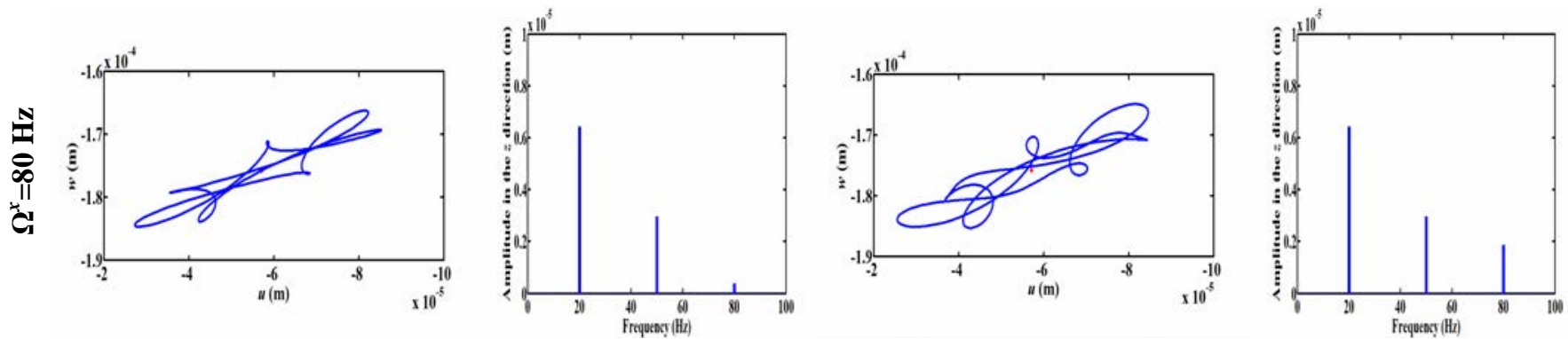

Figure 11. ORBITS AND THEIR $z$-FFTs AT THE DISK FOR FLEXIBLE BEARINGS AND BASE HARMONIC TRANSLATION $\left(Z_{0}=5 \times 10^{-5}\right.$ AND $\left.\Omega^{z}=50 \mathrm{~Hz}\right)$, COMBINED WITH TWO AMPLITUDES OF BASE HARMONIC ROTATIONS: $\omega^{x, a}=(a) 10^{-2} \mathrm{rad} / \mathrm{s}$, (b) $5 \times 10^{-2} \mathrm{rad} / \mathrm{s},\left(\Omega^{x}=20 \mathrm{~Hz}, 50 \mathrm{~Hz}\right.$ AND $\left.80 \mathrm{~Hz}\right)$

\section{REFERENCES}

[1] Rao, J. S., 1992, Rotordynamics, Wiley, New York.

[2] Lalanne, M., and Ferraris, G., 1998, Rotordynamics Prediction in Engineering, Wiley, Chichester.

[3] Berlioz, A., Der Hagopian, J., Dufour, R., and Draoui E., 1996, "Dynamic Behavior of a DrillString: Experimental Investigation of Lateral Instabilities," ASME J. Vibr. Acoust., 118(3), pp. 292-298.

[4] Nandi, A., and Neogy, S., 2005, "An Efficient Scheme for Stability Analysis of Finite Element Asymmetric Rotor Models in a Rotating Frame," Finite Elem. Anal. Des., 41(14), pp. 1343-1364.

[5] Samali, B., Kim, K. B., and Yang, J. N., 1986, "Random Vibration of Rotating Machines Under Earthquake Excitations," ASCE J. Eng. Mech., 112(6), pp. 550-565.

[6] Hori, Y., and Kato, T., 1990, "Earthquake-Induced Instability of a Rotor Supported by Oil Film Bearings," ASME J. Vibr. Acoust., 112(2), pp. 160-165.

[7] Bachelet, L., Driot, N., and Ferraris, G., 2006, "Rotor Under Seismic Excitation: A Spectral Approach," Proceedings of IFToMM 7th International Conference on Rotor Dynamics, Vienna, Austria, Sept. 25-28.

[8] Da Silva Tuckmantel, F. W., Cavalca, K. L., De Castro, H. F., Felscher, P., and Markert, R., 2011, "An Analysis on the Supporting Structure Representative Model in Rotating Systems," Proceedings of the Tenth International Conference on Vibration Problems, Prague, Czech Republic, Sept. 5-8.

[9] Kang, Y., Chang, Y.-P., Tsai, J.-W., Mu, L.-H., and Chang, Y.-F., 2000, "An Investigation in Stiffness Effects on Dynamics of Rotor-Bearing-Foundation Systems," J. Sound Vib., 231(2), pp. 343-374.

[10] Cavalca, K. L., Cavalcante, P. F., and Okabe, E. P., 2005, "An Investigation on the Influence of the Supporting Structure on the Dynamics of the Rotor
System," Mech. Syst. Signal Proc., 19(1), pp. 157-174.

[11] Duchemin, M., Berlioz, A., and Ferraris, G., 2006, "Dynamic Behavior and Stability of a Rotor Under Base Excitation," ASME J. Vibr. Acoust., 128(5), pp. 576-585.

[12] Driot, N., Lamarque, C. H., and Berlioz, A., 2006, "Theoretical and Experimental Analysis of a BaseExcited Rotor," ASME J. Comput. Nonlinear Dyn., 1(3), pp. 257-263.

[13] El-Saeidy, F. M. A., and Sticher, F., 2010, "Dynamics of a Rigid Rotor Linear/Nonlinear Bearings System Subject to Rotating Unbalance and Base Excitations. J. Vib. Control, 16(3), pp. 403-438.

[14] Das, A. S., Dutt, J. K., and Ray, K., 2010, “Active Vibration Control of Unbalanced Flexible RotorShaft Systems Parametrically Excited Due to Base Motion," Appl. Math. Model., 34(9), pp. 2353-2369.

[15] Frêne, J., Nicolas, D., Degueurce, B., Berthe, D., and Godet, M., 1997, Hydrodynamic Lubrication Bearings and Thrust Bearings, Elsevier Science, Amsterdam. 